\title{
Measurement of MOSFET LF Noise Under Large Signal RF Excitation
}

\author{
Arnoud P. van der Wel, Eric A. M. Klumperink and Bram Nauta \\ $M E S A+$ research institute, IC-Design group, University of Twente, Enschede, The \\ Netherlands. \\ a.p.vanderwel@utwente.nl \\ URL: icd.el.utwente.nl
}

\begin{abstract}
A new measurement technique is presented that allows measurement of MOSFET LF noise under large signal RF excitation. Measurements indicate that MOSFETS exhibit a reduction in LF noise when they are cycled from inversion to accummulation and that this reduction does not depend on the frequency of excitation for excitation frequencies of up to $3 \mathrm{GHz}$.

The measurement results are significant because MOSFET LF noise is important in the design of RF CMOS circuits such as oscillators and mixers, where large signal swings occur. Additionally, the measurement results give new insights into the LF noise generating mechanisms in MOSFETS.
\end{abstract}

\section{Introduction}

In RF CMOS designs, the LF noise performance of the devices is of critical importance as LF noise is often a limiting factor in the performance of the circuit. For example, close-in phase noise of GHz- range VCO's is dominated by LF device noise. The performance of downconversion mixers in zero- and low-IF systems is also limited by LF device noise. For correct design good models, substantiated by accurate measurements, are essential.

LF noise in MOSFETs is modelled as a biasindependent gate voltage source $(\Delta \mathrm{N}$ model) or as a bias-dependent gate voltage source $(\Delta \mu$ model). The n-channel devices are often seen to fit better to the $\Delta \mathrm{N}$ model, while p-channel devices often fit better to the $\Delta \mu$ model [2].

Current circuit simulator noise models based on [1] correctly account for such bias dependence but still always assume that LF noise of the device can be modelled as a memoryless function of the bias condition of the device. Models such as these are closely associated with the way most noise measurements are carried out: the bias voltage is kept constant during each measurement.

Recent measurements of MOSFET LF noise under large-signal excitation, where the bias voltage varies rapidly relative to the $\mathrm{LF}$ noise of interest, have shown that MOSFET LF noise is not only a function of the momentary bias of the device but also of the bias history of the device. Specifically, experiments show that significant LF noise reduction can be obtained when a MOSFET is rapidly cycled between strong inversion and accumulation. [3, 4, 5, 6, 7]. These measurement were done at excitation frequencies in the $\mathrm{kHz}$ and low $\mathrm{MHz}$ region. This paper is the first to address the problem of LF noise measurement under large signal excitation at RF. These operating conditions are very similar to what the devices might encounter in real RF CMOS circuits such as oscillators and mixers.

A new measurement method will be presented. It excites the MOSFET with a large-signal sinewave while measuring the LF noise. Using this method we are able to perform accurate measurements with an excitation frequency of up to $500 \mathrm{MHz}$, and up to $3 \mathrm{GHz}$ with a somewhat larger error margin.

This paper is organised as follows. Section 2 discusses the problem of LF noise characterisation under large signal RF excitation and introduces a new measurement technique to solve this problem. Section 3 presents and discusses the results obtained with the technique. Section 4 ends with conclusions.

\section{RF measurement method}

The aim of this paper is to examine the LF noise of MOS transistors under large signal excitation, but now at high excitation frequencies. As mentioned in the previous section, large signal measurements until now were done with a square-wave at frequencies of at most $1 \mathrm{MHz}$. At those frequencies this is a quite feasible method. However, generating a well-behaved square-wave at RF is much more difficult than generating a sinewave at the same frequency as fast square waves place extreme demands on the bandwidth of the circuitry. Moreover, Typical square-wave signal generators are not designed to produce a low-noise square-wave. (Equivalent noise resistance values as high as $1 \mathrm{M} \Omega$ are no exception for $50 \Omega$ pulse-generators.) In contrast, low noise sinewave generators for RF frequencies are readily available, so this is a much more suitable choice for RF measurements.

While using a sine-wave instead of a square-wave is much more feasible at RF, it makes interpretation of the measurement results more difficult: 
It is relatively easy to account for systematic effects when the device is biased by a square-wave: the device essentially resides in only two biasing points, one with significant LF noise (on-state in strong inversion) and the other with negligible noise contribution (off-state in weak inversion or accumulation) [6]. Like this, the square-wave bias noise measurement can easily be compared to a DCbias noise measurement.

When, on the other hand, the device is excited by a sine wave, it passes through many biasing points each period, so comparison of a sine-wave excited noise measurement to a DC-bias noise measurement is not straightforward.

The solution to this problem is found by realizing that the MOS device only contributes significant noise during the time the gate source voltage is above threshold (and Id $\neq 0$ ). Also, we know from switched biasing experiments [7], that the device noise reduction is a function of the minimum off-voltage. Hence, we want to vary the minimum off-voltage while keeping the waveform above threshold the same: The waveform we want is shown in fig. 1 .

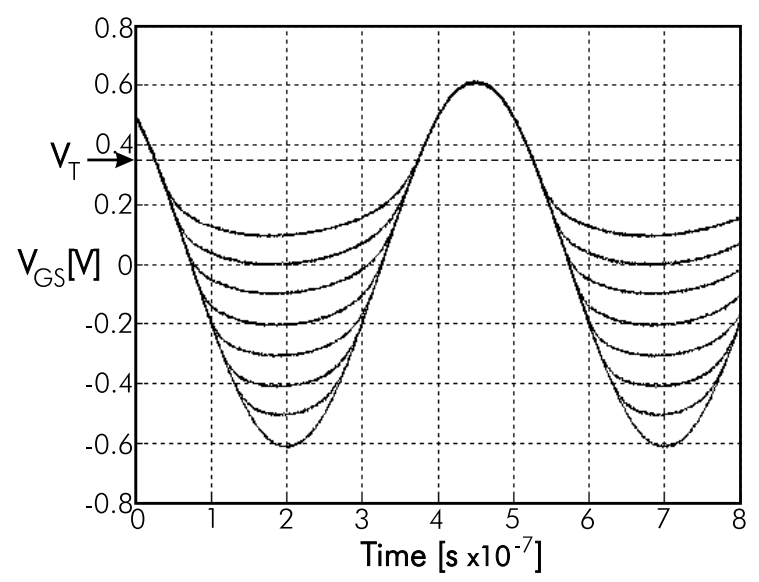

Figure 1. Excitation voltage waveform with a variable minimum voltage

In fig. 1, the threshold voltage of the device under test is also shown. As long as the waveform above threshold does not change, neither does the DC drain current of the device, and simulators using existing MOSFET noise models predict that the LF noise does not change either.

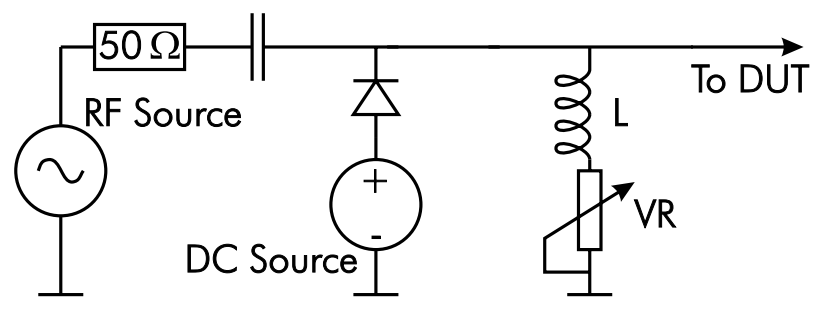

Figure 2. Circuit to produce the waveform of fig. 1
The circuit we use to produce such a waveform is shown in fig. 2. The diode in this circuit conducts during the negative half-period of the driving sinewave, limiting the negative excursion of the waveform. An RF Choke ' $L$ ' in combination with a variable resistor ensures that the positive half of the waveform does not shift as the minimum off-voltage is varied. The variable resistor needs to be adjusted only once.

We apply this waveform to the gates of the devices under test (see fig. 3). Two identical DUTs are driven inphase. This allows us to use the common mode rejection of a differential probe to filter out the RF driving signal and measure the LF noise of the devices. A low pass filter at the drains (not shown; $f_{c}=100 \mathrm{kHz}$ ) helps to keep the drain voltage of the devices constant during the measurement.

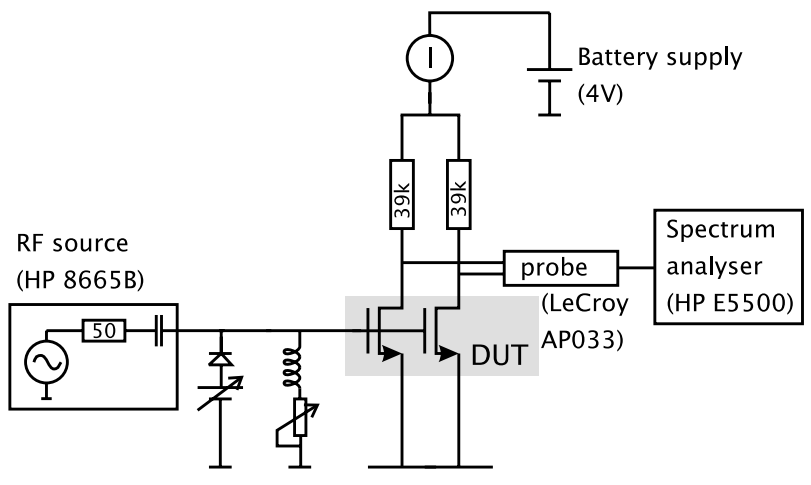

Figure 3. The complete measurement setup

This measurement is carried out at $2 \mathrm{MHz}$. The variable resistor is adjusted so that the top part of the sinewave is not influenced by a variation in the minimum offvoltage, as witnessed by the fact that the DC drain current of the devices does not change as the minimum offvoltage is varied. For additional verification that varying the minimum off-voltage does not influence the effective transconductance of the devices, a small pilot tone (150 $\mu \mathrm{V}$ at $5 \mathrm{kHz}$ ) is injected into the gate of one of the devices under test and its amplitude at the drain is measured. The time-average transconductance of the devices was indeed found to be independent of the minimum off-voltage.

We can now measure the LF noise of the devices as a function of the minimum off-voltage of the driving waveform.

Having carried out this varying off-voltage measurement we can proceed to RF sinewave measurements. These are carried out with an unmodified sine wave driving the gates of the DUTs. (The full sinewave with an amplitude of $600 \mathrm{mV}$ appears at the gate of the DUTs.) As the frequency of excitation is varied, the bias voltage trajectory of the devices does not change, and neither the average drain current nor the effective transconductance of the device changes. Again, injecting a pilot tone and measuring its amplitude at the drain verifies that this assumption is correct. 


\section{To sum up the two-step measurement method:}

1. At an excitation frequency of $2 \mathrm{MHz}$, we demonstrate that the LF noise of the devices is reduced if the device is cycled to accumulation.

2. We then show that the LF noise remains low for excitation frequencies of up to $3 \mathrm{GHz}$.

\section{RF measurement results}

Measurements were carried out on six pairs of devices from the same wafer. Devices were contacted using a Cascade wafer prober and Picoprobe coaxial probes. The RF signal path is a $50 \Omega$ line terminated at the DUTs. The devices were n-channel devices from a $0.35 \mu \mathrm{m}$ process: $\mathrm{T}_{o x}=7.5 \mathrm{~nm}, \mathrm{~V}_{T}=0.35 \mathrm{~V}, \mathrm{~W} / \mathrm{L}=50 / 0.35 \mu \mathrm{m} . \mathrm{V}_{D}=2 \mathrm{~V}$, Amplitude of driving signal $=600 \mathrm{mV}$ at gate. Average current through each device is $50 \mu \mathrm{A}$ for all measurements.

The results are shown in fig 4 and fig 5.

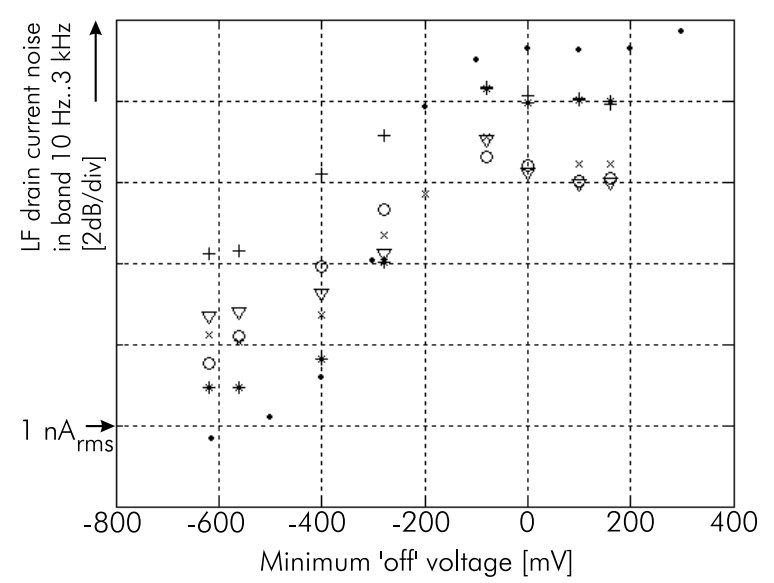

Figure 4. Measurement results at $2 \mathrm{MHz}$

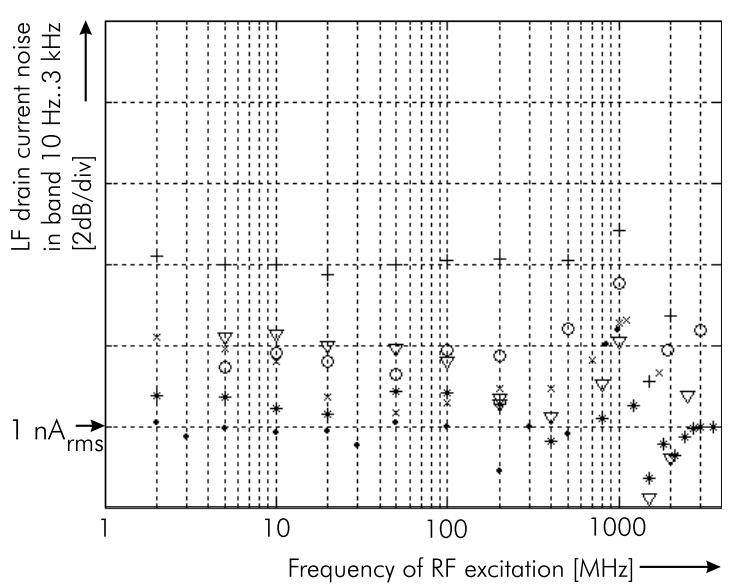

Figure 5. Measurement results at higher frequencies
For each measurement, the LF noise spectrum is measured using a spectrum analyser. The spectrum is integrated from $10 \mathrm{~Hz}$ to $3 \mathrm{kHz}$, and the integrated noise is plotted in fig. 4 and fig. 5. For reference, $1 \mathrm{nA}_{R M S}$ drain current noise (in the same bandwidth) is shown in both figures.

All devices show the same trend.

Fig. 4 shows that as the minimum off-voltage is reduced, without changing the effective transconductance of the device, the LF noise of the device is reduced. The reduction (just as the absolute noise level) shows spread from device to device. It is in the range of 4-10 dB. This is in accordance with [8] where a large spread in the noise reduction from device to device was also observed.

Fig. 5 shows that (when driving the DUTs with an unmodified sinewave, ) as the frequency of excitation is increased, the noise reduction observed at $2 \mathrm{MHz}$ is retained. The accuracy of the measurements is estimated at better than $+/-0.5 \mathrm{~dB}$ at frequencies up to $500 \mathrm{MHz}$, and slightly less for higher frequencies.

\section{Conclusions}

Using a novel measurement technique, LF noise in nchannel MOSFETs under large signal excitation at RF frequencies has been measured. These conditions are very similar to what the devices may encounter in real-life circuits such as mixers and oscillators, and hence, study of LF noise under these conditions is relevant.

Using a two-step measurement method, we have shown that:

- Cycling the device to accumulation at $2 \mathrm{MHz}$ reduces its LF noise by up to $10 \mathrm{~dB}$, where circuit simulators using current models predict no change.

- The noise reduction observed by cycling to accumulation is essentially independent of the frequency of excitation for excitation frequencies of up to at least $3 \mathrm{GHz}$.

\section{Acknowledgements}

The authors wish to thank Philips Research for providing test structures. We also wish to thank Wouter Groothedde, Gerard Wienk and Sander Smits for contributions to this work. This work is supported by the Technology Foundation (STW), Applied Science division of NWO, and the Technology Programme of the Netherlands Ministry of Economic Affairs.

\section{References}

[1] Kwok K. Hung, Ping K. Ko, Chenming $\mathrm{Hu}$ and Yiu C. Cheng, "A Unified Model for the Flicker Noise in Metal-Oxide-Semiconductor Field-Effect Transistors", IEEE Trans. Electron Devices, Vol. 37, No. 3, March 1990, pp. 654-665. 
[2] L.K.J. Vandamme, X.Li, and D. Rigaud, "1/f Noise in MOS devices, mobility or number fluctuations?", IEEE Trans. Electron Devices, Vol. 41, No. 11, Nov. 1994, pp. 19361944.

[3] I. Bloom and Y. Nemirovsky, "1/f noise reduction of metal oxide semiconductor transistors by cycling from inversion to accumulation," Applied Physics Letters, Vol. 58, No. 15, April 1991, pp. 1664-1666.

[4] B. Dierickx and E. Simoen, "The decrease of Random Telegraph Signal noise in Metal-Oxide-Semiconductor FieldEffect Transistors when cycled from inversion to accumulation",Journal of Applied Physics, Vol. 71, No. 4, 15 February 1992, pp. 2028-2029.

[5] S. L. J. Gierkink, E. A. M. Klumperink, A. P. van der Wel, G. Hoogzaad, A. J. M. van Tuijl and B. Nauta, "Intrinsic 1/f Device Noise Reduction and its Effect on Phase Noise in CMOS Ring Oscillators", IEEE Journal of Solid-State Circuits, Vol. 34, No. 7, July 1999, pp. 1022-1025.

[6] E. A. M. Klumperink, S. L. J. Gierkink, A. P. van der Wel, and B. Nauta, "Reducing MOSFET 1/f Noise and Power Consumption by Switched Biasing", IEEE Journal of SolidState Circuits, Vol. 35, No. 7, July 2000, pp. 994-1001.

[7] A. P. van der Wel, E. A. M. Klumperink, S. L. J. Gierkink, R. F. Wassenaar, and H. Wallinga, "MOSFET 1/f noise measurement under switched bias conditions", IEEE Electron Device Letters, Vol. 21, No. 1, Jan. 2000, pp. 43-46.

[8] A. P. van der Wel, E. A. M. Klumperink and B. Nauta, "Effect of switched biasing on 1/f noise and random telegraph signals in deep-submicron MOSFETs", Electronics Letters, Vol. 37, No. 1, 4th Jan. 2001, pp. 55-56. 